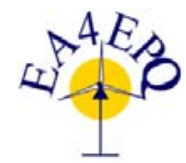

International Conference on Renewable Energies and Power Quality (ICREPQ’13)

Bilbao (Spain), 20 ${ }^{\text {th }}$ to $22^{\text {th }}$ March, 2013

Renewable Energy and Power Quality. Tournal (RE\&PQJ)

ISSN 2172-038 X, No.11, March 2013

\title{
Filtering Techniques: An historical overview and summary of current status
}

\author{
J. Lazaro ${ }^{2}$, J.F. Miñambres ${ }^{1}$, M.A. Zorrozua ${ }^{1}$, \\ ${ }^{1}$ Department of Electrical Engineering \\ ${ }^{2}$ Department of Applied Mathematics \\ E.T.S.I., University of the Basque Country (UPV/EHU) \\ Alda. Urquijo s/n, 48013 Bilbao (Spain) \\ Phone/Fax number:+0034 946014150 / +0034 94 6014200, e-mail: jorge.lazaro@ehu.es
}

\begin{abstract}
A thoroughly overview and summary of current status is provided in order to fully understand the pros and cons of the different filtering methods used in power system quality assessment. The aim of this paper is to shorten the time needed in order to understand the different types of existing algorithms along the limitations and burdens imposed by their mathematical foundations.
\end{abstract}

\section{Key words}

Filtering techniques, Harmonic analysis, Spectral analysis.

\section{Introduction}

Digital signal processing (DSP) has become an ineludible step when trying to ascertain the harmonic spectrum within almost any electrical signal characteristic of a power system. DSP is usually carried away by an algorithm that performs mathematical operations on a sampled electrical signal to reduce or enhance certain aspects of that signal and also know its harmonic composition. This harmonic spectrum will be of the greatest importance in order to make critical decisions when operating the power system. Protective relaying will make also an extensive use of that spectrum in order to optimize the behavior of the system against faulty conditions.

The algorithm in charge of the digital signal processing analysis is also known as digital filter. It usually features some specific mathematical techniques and operations fed with the samples from the signal.

As digital equipments are becoming ubiquitous in power systems, digital filters have to be improved all the time to cope with the different characteristics featured by each device. Such improvements usually have to do with accuracy, speed and stability issues arising due to the ever growing variety and complexity of the conditions faced along the operation of the power system.
Electrical signals within a power system can change dramatically between the steady state and transient states caused by normal or abnormal conditions taking place within the grid. Normal conditions are usually associated to common maneuvers such as connecting, switching and shutting down any subgrid of the system for maintenance or replacement jobs. Abnormal conditions are mostly caused by faulty events affecting any component of the grid.

Calculation speed and computational burden are two characteristics of a filtering algorithm that are differently approached depending on the time constraints of the particular problem addressed in the design. Two main fields of applications are considered here: on-line and off-line problems.

On-line problems are all the issues related to protection and control of the power system. Here, speed is critical and the computational burden often collides with limitations inherent to the DSP in charge of the calculations.

Off-line problems are usually related to power quality in a wide variety of areas ranging from fault location to the calculation of THD and other parameters used to "measure" the quality in the performance of the power system.

\section{Response of a digital filter: conditioning factors}

In the steady state, a typical signal may consist of a set of harmonics and interharmonics plus an offset and noise. The presence of interharmonics is increasingly growing in the power system due the spreading use of power electronics within the grid. Arcing loads, ripple controls, static converters and variable load electric drives are 
among the main sources of interharmonics in current power systems.

Noise is frequently neglected in many papers presenting new filtering algorithms despite its critical influence in the output when stability issues arise. In transient states, along the aforementioned components, there may appear aperiodic sub-signals such as exponential dc-offsets, saturation inrush currents, etc, causing convergence problems in DFT based algorithms among others.

Stability and convergence issues frequently render useless, or at least seriously hinder both its accuracy and speed, many algorithms that perform perfectly in the absence of noise.

\section{Characteristics of ideal digital filters}

The characteristics that must be fulfilled by a digital filter depend on its field of application. Table 1 shows the expected characteristics according to its two main fields of application mentioned in Section 1.

Table I. - Characteristics of ideal digital filters

\begin{tabular}{|l|c|c|}
\cline { 2 - 3 } \multicolumn{1}{c|}{} & OFF & ON \\
LINE & LINE \\
\hline Accuracy & $\mathrm{X}$ & $\mathrm{X}$ \\
\hline Fast response & & $\mathrm{X}$ \\
\hline Low computational burden & & $\mathrm{X}$ \\
\hline Low sampling rate & & $\mathrm{X}$ \\
\hline Stability against noise & $\mathrm{X}$ & $\mathrm{X}$ \\
\hline System state independent & & $\mathrm{X}$ \\
\hline
\end{tabular}

The requirements in 'on line' applications are more demanding due to the time constraints.

\section{Main types of filtering techniques}

From the different publications [1]-[12] in the field along the last years, filtering algorithms may be grouped into three main categories:

- FA1: filtering algorithms based on matricial methods such as LES (Least Error Squares). All of the characteristic in the analysed signal must be explicitly considered for the mathematical model. Matrices involved in the calculations are often bad conditioned and, thus, leading to severe stability problems. To strengthen these methods against errors, large sets of data are needed resulting in oversized matrices whose manipulation collides with the admissible computational capabilities of the DSP.

- FA2: algorithms emulating mimic filters for a preset range of characteristics of the electrical signal. A mimic filter can be implemented in analog or digital circuitry, and it is designed and tuned to remove dc offset or other noise from an input signal using a pseudodifferentiation technique. The main problem here is that to properly tune the filter, some characteristics of the analysed signal must be preset in advance. This makes its accuracy depend on how close are the preset parameters to the real ones.

- FA3: modified versions of the DFT (Discrete Fourier Transform) designed in order to cope with non harmonic components. Although the mathematical foundations of the DFT make it very stable against noise, further modifications of the algorithm usually result in serious stability issues which, if not properly addressed, render these algorithms hardly usable against noisy signals.

\section{Comparison of filtering techniques}

Theoretical signals are signals without noise and with a number and type of components known. In general, it can be said that the behaviour of all the proposed filtering techniques is satisfactory when they are used to analyze theoretical signals. However, this is not sufficient to guarantee that they are able to analyze the actual signals present in an electric power system. Nonetheless, the validation process of a significant number of filtering techniques proposed in the last years only include theoretical signals as input signals. By this reason, some of the proposed filtering techniques are impeccable from a purely mathematical point of view but they are not suitable to be used in practical applications.

To illustrate this problematic, Section 5 presents the analysis and comparison of different digital filters proposed in the last years and belonging to the categories defined in Section 4.

\section{A. Filter algorithms belonging to FA1 category}

LES based algorithms are usually carried out by means of a matrix equation of the form $I=M \cdot P$ in which the samples vector $(I)$ and the time information $(M)$ allow us to obtain all the unknowns $(P)$ as follows:

$$
P=\left(M^{T} \cdot M\right)^{-1} \cdot M^{T} \cdot I
$$

For a simple signal like:

$$
y(t)=C \cdot e^{-t / \tau}+A \cdot \cos \left(\frac{\pi}{10} \cdot t+\frac{\alpha \cdot \pi}{180}\right)
$$

where $C=100, A=100, \tau=100 m s$ and $\alpha=10$, the matrix $M^{T} \cdot M$ for 4 samples looks as follows:

$\left(\begin{array}{cccc}3.72162 & 0.920225 & 0.00297597 & 3.85679 \\ 0.920225 & 0.278379 & 0.000903058 & 0.966076 \\ 0.00297597 & 0.000903058 & 2.92969 \times 10^{-6} & 0.003125 \\ 3.85679 & 0.966076 & 0.003125 & 4.0\end{array}\right)$


The heavily unbalanced entries in this matrix result in a bad conditioning issue which is easily understandable from any criterium considered to calculate the conditioning number for $M^{T} \cdot M$ :

$$
\begin{aligned}
& C N D_{1}=\frac{\lambda_{\max }}{\lambda_{\min }}=1.74299 \times 10^{13} \\
& C N D_{2}=\frac{\left(M^{T} M\right)_{i, j}^{\max }}{\left(M^{T} M\right)_{i, j}^{\min }}==2.0253 \times 10^{12} \\
& C N D_{3}=\frac{\left\|M^{T} M\right\|}{\left\|\left(M^{T} M\right)^{-1}\right\|}=1.74583 \times 10^{13}
\end{aligned}
$$

These big conditioning numbers reveal an stability problem that turns into great errors in the output when small perturbations ( $0.1 \%$ of $\mathrm{A})$ affect the input data as it is shown in Table II.

Table II. - Errors in LES output due to perturbations in input data.

\begin{tabular}{|c|c|c|c|c|}
\cline { 2 - 5 } \multicolumn{1}{c|}{} & $A$ & $\alpha\left(^{\circ}\right)$ & $\mathrm{C}$ & $\tau(\mathrm{ms})$ \\
\hline$y(t)$ & 100 & 10 & 100 & 100 \\
\hline LES $y(t)$ & 99.9 & 10.0 & 100.1 & 100.8 \\
\hline $\begin{array}{c}\text { LES } y(t) \\
\text { with noise }\end{array}$ & 165.6 & 46.9 & 85.3 & -2.7 \\
\hline Error(\%) & 65.6 & 36.8 & 14.6 & 102.7 \\
\hline
\end{tabular}

\section{B. Filter algorithms belonging to FA2 category}

The basis of the different mimic filters is the methodology defined in [8]. This methodology is not really a filtering technique. It is a methodology to eliminate the decaying dc offset present in electrical transient signals. In addition, the accurate elimination of this non-periodic component requires the knowledge of its corresponding time constant value.

Consequently, the mimic technique must be complemented with the use of two additional algorithms. A first algorithm for estimating the time constant value and a latest algorithm for estimating the periodic component value (amplitude and angle) that is being calculated.

By this reason, the behaviour of a filter belonging to the FA2 category is directly determined by the behaviour of the filter used in its last step to estimate the periodic component [9]. Usually, this last algorithm belongs to the FA3 category because algorithms belonging to the FA1 category do not require the previous elimination of decaying dc-offset. In any case, the behaviour of the filters belonging to the FA1 and FA2 categories is analysed in the corresponding subsections A and C.

\section{Filter algorithms belonging to FA3 category}

These algorithms are based on the DFT. The results provided by the DFT are very stable against noise but its output is oscillatory when it is used to analyze transient signals that include non-periodic components. The DFT modifications are proposed with the aim of improving the DFT behavior against transient signals. These modifications result in new algorithms that must be tested in the presence of both noise and non-periodic components in the analyzed signal.

Below is the comparison between the results provided by some of main algorithms belonging to FA3 category and the results provided by the DFT.

As is well known, digital filters provide two outputs: amplitude and angle. The problematic mentioned above affects the results of the estimation of both. Fig. 1 shows the amplitude and angle estimation output corresponding to DFT and [1] when they are used to estimate the fundamental component of a transient fault signal in the absence of noise.

As can be seen, [1] provides accuracy results and DFT provides results affected by significant oscillations due to non-periodic components present in the signal being filtered.
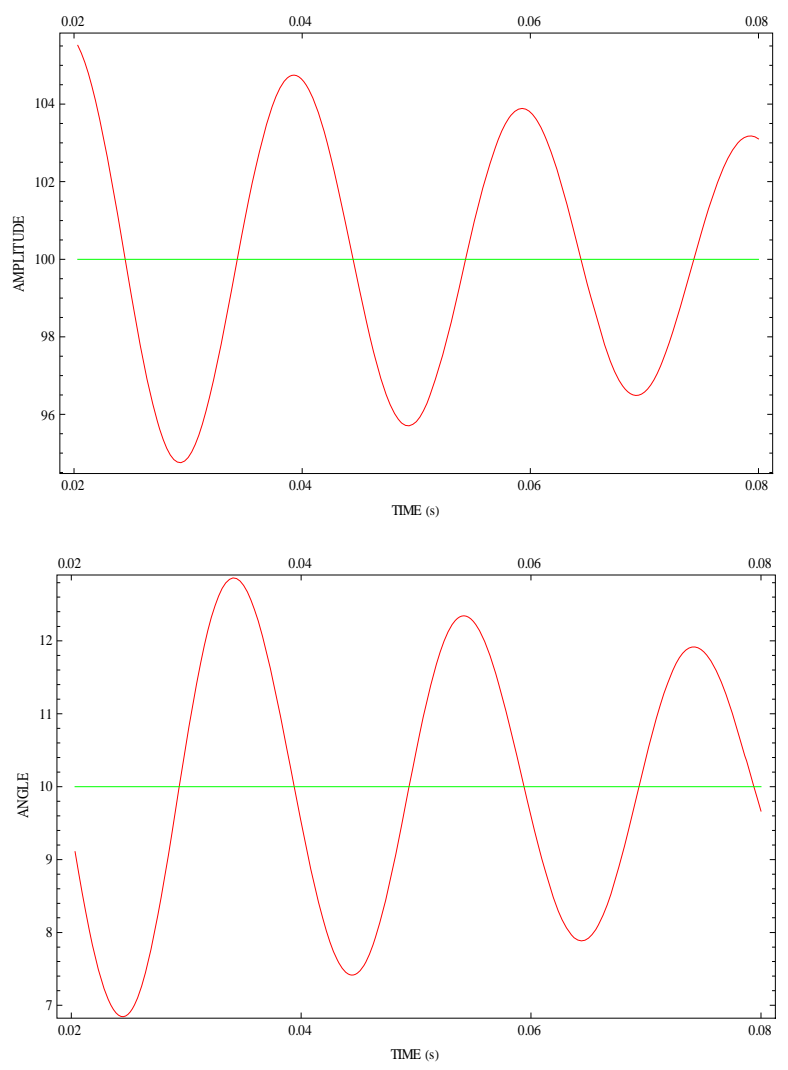

Fig. 1. Output (DFT y [1]) for the fundamental component in a faulty signal in the absence of noise.

However, Fig. 2 shows that the presence of a minimum noise $(0.1 \%$ respect to the fundamental component amplitude) modifies significantly the output of [1]. The improvement disappears and the response is even worse than the corresponding to the DFT. 

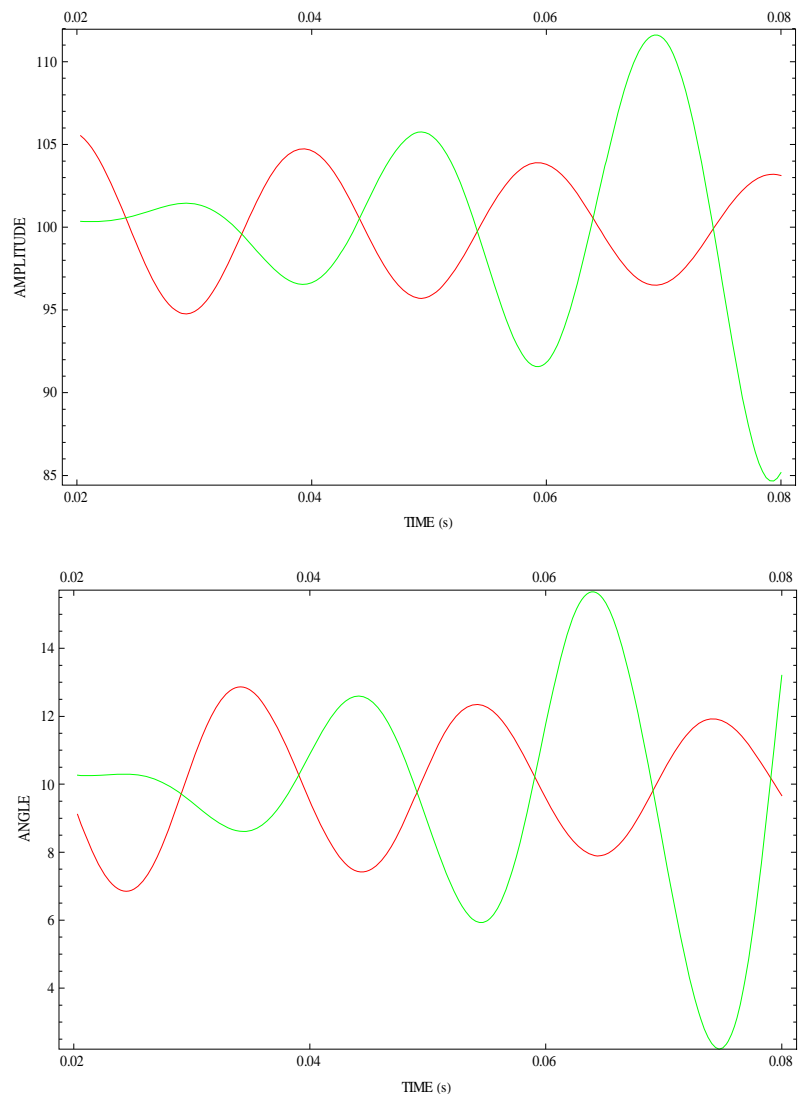

Fig. 2. Output (DFT y [1]) for the fundamental component in a faulty signal with noise.
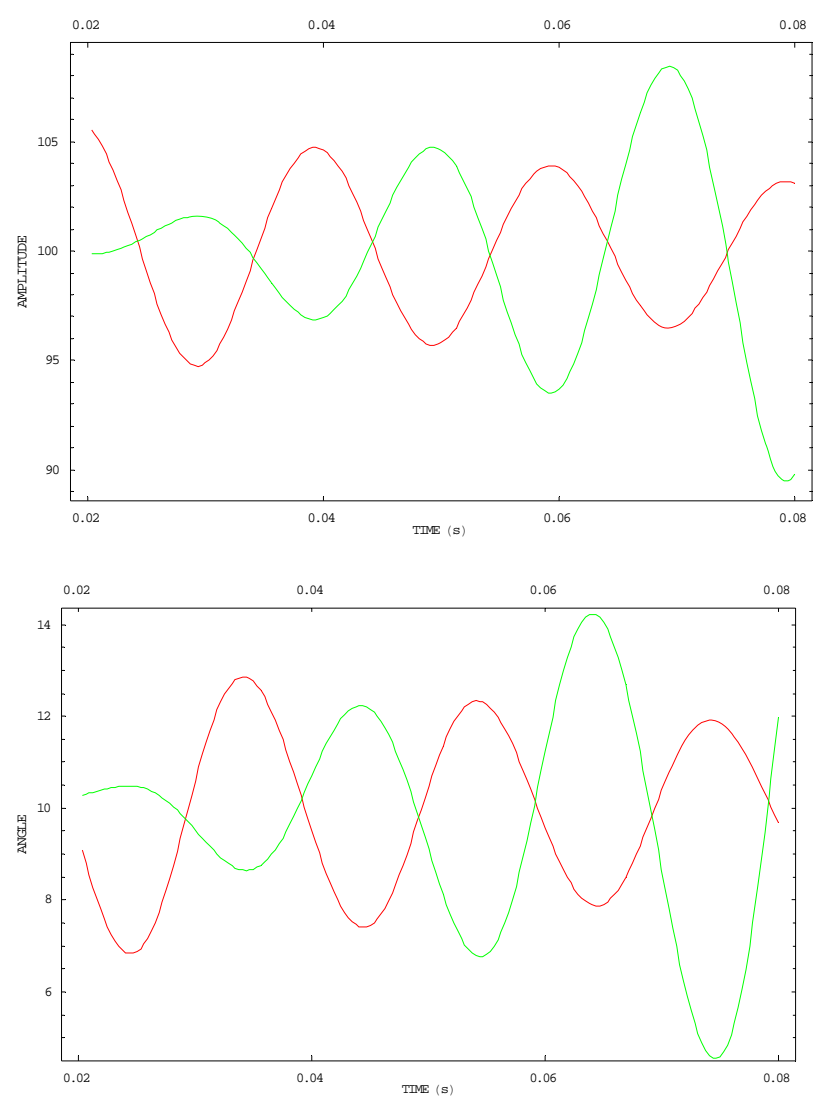

Fig. 3. Output (DFT y [10]) for the fundamental component in a faulty signal with noise.
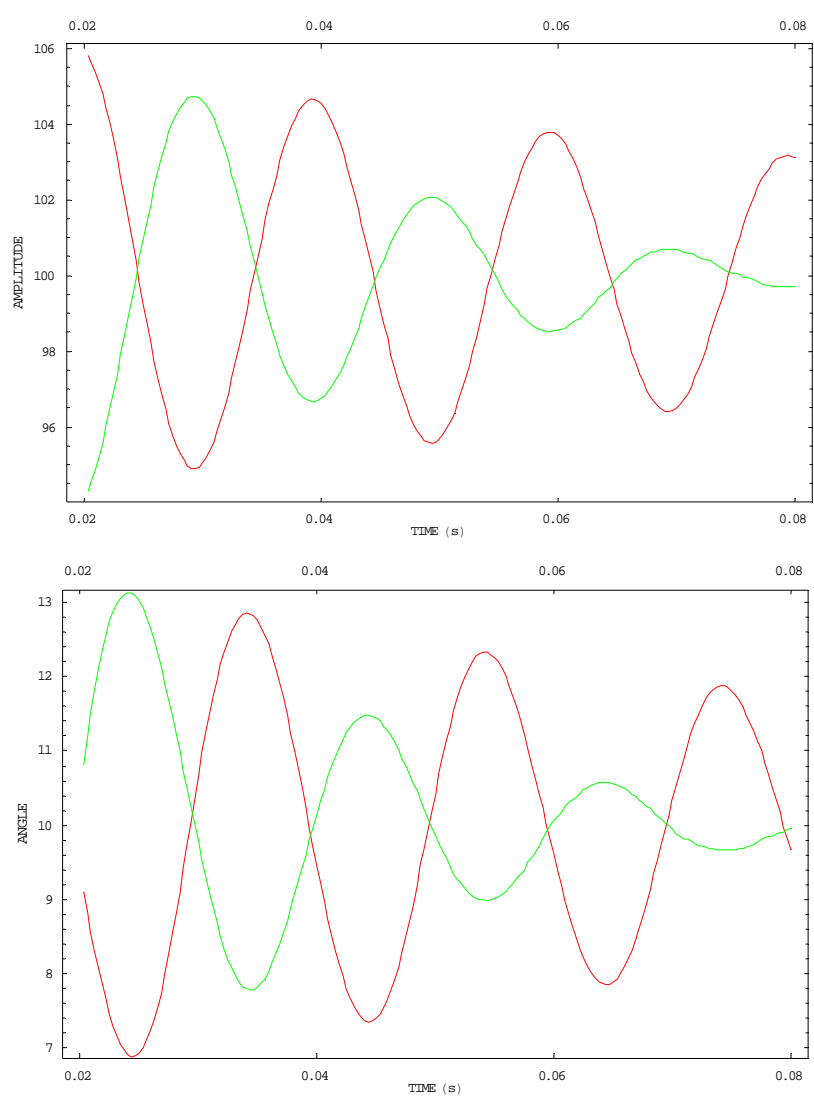

Fig. 4. Output (DFT y [11]) for the fundamental component in a faulty signal with noise.
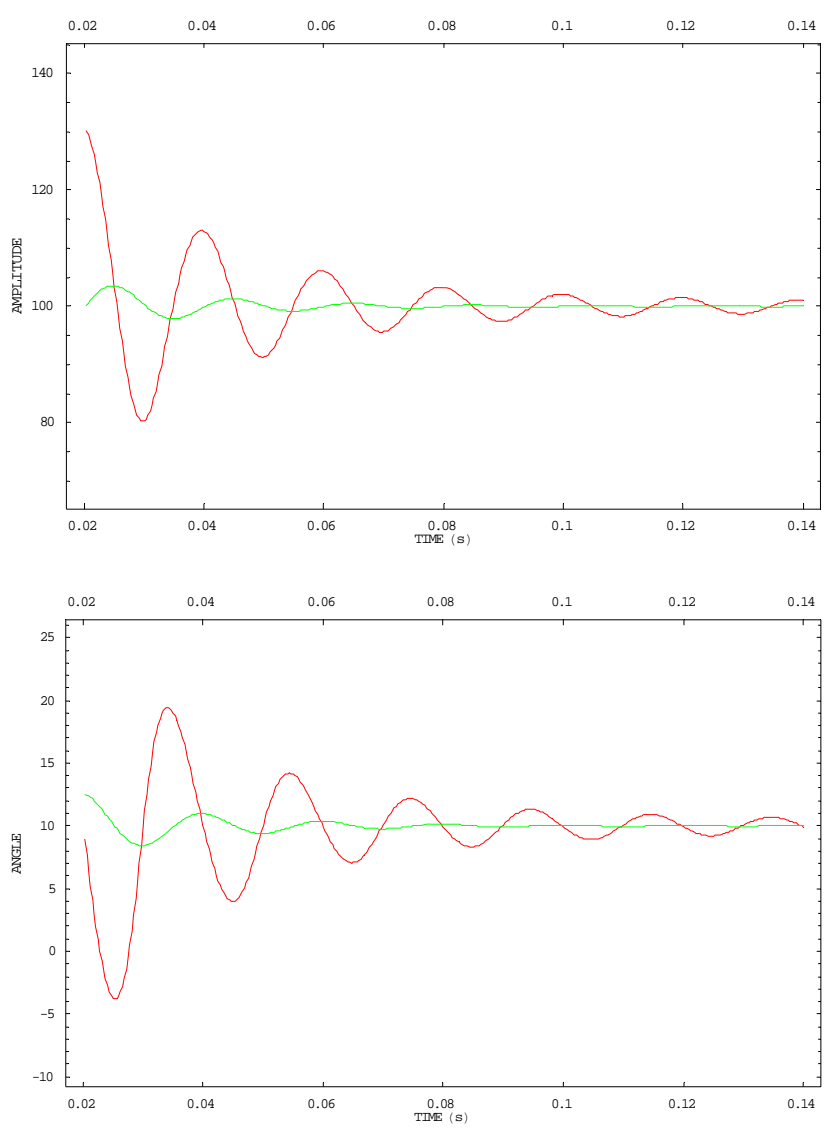

Fig. 5. Output (DFT y [12]) for the fundamental component in a faulty signal with noise. 
In the same way, the comparison between DFT and [10] is presented in Fig. 3 (noise of $0.1 \%$ ) and the comparison between DFT and [11] is presented in Fig. 4 (noise of 1\%). In both cases, the outputs corresponding to the analysis of faulty signals without noise have been omitted due to that they are similar to Fig.1.

By contrast, algorithms that respond well in the presence of noise are suitable for use in any application. This is the case of [12]. Fig. 5 shows the comparison between DFT and [12] (noise of 1\%).

\section{Conclusions}

The main goal in this paper was to provide a comparative analysis of filtering algorithms for electrical signals.

In order to get a better insight into the performances of the different methods proposed along the last years, the evaluated algorithms have been tested under very demanding conditions to match the requirements usually found in electrical power systems.

From the results obtained in the series of tests carried out with the considered methods the following conclusions should be taken into account:

- FA1 and FA2 methods are suitable for off-line applications but do not match the requirements demanded by devices working on line such as protection relays. This is due to both the big computational loads involved and the need for supplementary algorithms (to determine $\tau$ for a proper calibration in FA2 or to distinguish between prefault and fault conditions in FA1).

- FA3 perform well in both off-line and on-line applications as they do not imply heavy computational burdens for the DSPs (digital signal processors) carrying out the calculations. These methods do not need either supplementary algorithms in order to discriminate steady and transient states.

Noise is a very important factor to be taken into account in any real time application. Noisy components are always present in real electrical signals (ADCs, magnetic coupling between PCBs, measurement errors...) and are frequently involved with stability issues that render useless many filtering algorithms otherwise well mathematically founded and performing correctly against "clean” signals.

- FA1 methods do not perform well against noisy signals due to bad conditioned matrices resulting in outputs that are heavily affected by the slightest errors in the analysed signals.

- FA3 methods usually involve ratios between small quantities ([1],[10],[11]) that are dramatically unstabilized by little noisy components. Though, when properly designed to avoid those issues ([12]), these algorithms can perform in a very stable way even in the presence of heavily distorted signals.

\section{References}

[1] J-C. Gu, S-L Yu, "Removal of DC offset in current and voltage signals using Fourier filter algorithm”, IEEE Transactions on Power Delivery, Vol. 15, No.1, pp. 73-79. January 2000.

[2] E.O. Schweitzer and D. Hou "Filtering for protective relays”, WESCANEX'93 Conference Proceedings, IEEE, , pp. 15-23, 1993

[3] O. Musa and A. Aksamovic "Comparative analysis of algorithms for elimination of exponentially decaying DC component”, IWSSIP 2012, Conference Publications, pp. 362-367, April 2012

[4] K.M. Silva and B.F. Küser "On combining waveletbased designed filters and an adaptive mimic filter for phasor estimation in digital relaying”, Electric Power Systems Research, Vol. 92, pp. 60-72, 2012

[5] J. Barros, R.I. Diego and M. de Apraiz “Applications of wavelets in electric power quality: Voltage events”, Electric Power Systems Research, Vol. 88, pp. 130-136, 2012

[6] J. Lázaro, J.F. Miñambres, M.A. Zorrozua, B. Larrea, M. Sánchez and I. Antiza "New quick-convergence invariant digital filter for phasor estimation”, Electric Power Systems Research, Vol. 79, no. 5, pp. 705713, May 2009

[7] W. M. Al-Hasawi and K. M. El-Naggar "New digital filter for unbalance distorted current and voltage estimation in power systems", Electric Power Systems Research, Vol. 78, pp. 1290-1301, 2008

[8] G. Benmouyal , "Removal of DC-offset in current waveforms using digital mimic filtering”, IEEE Transactions on Power Delivery, Vol. 10, No. 2, April 1995, pp. 621-630.

[9] CH. S. Yu , "A discrete Fourier transform-based adaptive mimic phasor estimator for distance relaying applications", IEEE Transactions on Power Delivery, Vol. 21, No. 4, October 2006, pp. 18361846.

[10] T.S. Sidhu, X. Zhang, F. Albasri, M.S. Sachdev, "Discrete-Fourier-transform-based technique for removal of decaying DC offset from phasor estimates”, IEE Proc.Generation, Transmission and Distribution, Vol. 150, No. 6, November 2003, pp.745-752.

[11] Y. Guo, M. Kezunovic, D. Chen, "Simplified algorithms for removal of the effect of exponentially decaying DC-offset on the Fourier algorithm”, IEEE Transactions on Power Delivery, Vol. 18, No. 3, July 2003, pp. 711-717.

[12] J. Lázaro, J.F. Miñambres, M.A. Zorrozua, B. Larrea, M. Sánchez and I. Antiza "New quickconvergence invariant digital filter for phasor estimation”, Electric Power Systems Research, Vol. 79, no. 5, pp. 705-713, May 2009 